\title{
Point-of-care biosensors in medicine: a brief overview of our achievements in this field based on the conducted research in EMRI (endocrinology and metabolism research Institute of Tehran University of medical sciences) over the past fourteen years
}

\author{
Kobra Omidfar $^{1,2} \cdot$ Anita Ahmadi $^{1,2} \cdot$ Leila Syedmoradi $^{1,2} \cdot$ Seyyed Mehdi Khoshfetrat ${ }^{1,2} \cdot$ Bagher Larijani $^{2}$
}

Received: 22 September 2020 / Accepted: 19 October 2020

(C) Springer Nature Switzerland AG 2020

\begin{abstract}
The growing demand of diagnostic tools with enhanced analytical characteristics in term of sensitivity, selectivity, and low response time has encouraged researches to conduct their research towards development of point-of-care (POC) biosensors. POC diagnostic devices are powerful tools for detection, diagnosis, and monitoring of diseases at its initial stage. The above characteristics encouraged us to conduct active multidisciplinary and collaborative research oriented towards the design and development of POC sensing systems. Here, we present a brief overview of our recent achievement in the field of biomedical POC devices implemented in paper based microfluidic and screen printing electrodes and discuss the critical limitations that need to be surmounted to facilitate their translation into clinical practice in the future.
\end{abstract}

Keywords Point of care testing $\cdot$ Healthcare system $\cdot$ Biosensor $\cdot$ Nano biosensor

\section{Introduction}

Development of effective diagnostic tools for early detection of clinical biomarkers in body fluids are of great importance, both in detecting disease and physiological signatures that are predictive of potential disease states as early as possible.

Although current sensing methods are capable of accurate and specific detection of biomarkers, they are considered unsatisfactory to meet the triple limitations, inherent to biomarker determination, of rapid, low-concentration and inexpensive measurement. These drawbacks were essentially changed by point-of-care biosensor devices. The integration of nanomaterials in point of care testings (POCT) proposes the opportunity of realizing portable, easy to use, cost effective,

Kobra Omidfar

omidfar@tums.ac.ir

1 Biosensor Research Center, Endocrinology and Metabolism Molecular-Cellular Sciences Institute, Tehran University of Medical Sciences, 14395/1179, Tehran, I.R., Iran

2 Endocrinology and Metabolism Research Center, Endocrinology and Metabolism Research Institute, Tehran University of Medical Sciences, Tehran, Iran and miniaturized analytical devices [1]. Such devices enable early disease monitoring and diagnosis, before outbreaks, at the time and place of patient care. POC devices are now becoming popular with interesting applications in the fields of personalized medicine as it can recognize different biomarkers based diseases. These devices were commercialized to diagnose and monitor various disorders such as diabetes, cardiovascular disease, cancer, and infectious diseases [2]. Additionally, the coronavirus disease 2019 (COVID-19) pandemic which affected millions of people around the world, again demonstrates the significance of early detection of specific clinical biomarkers for healthcare monitoring system in global, and for risk prevention of further spread [3]. In Fig. 1a, the components of POC biosensing systems have been shown.

During the past fourteen years, most of our research projects focused on the development and application of POC diagnostic devices for the highly sensitive and selective detection of clinical analytes including metabolic, nucleic acid, and protein biomarkers in the Biosensor Research Center of EMRI [1]. Recently, paper has been applied as a simple and low-cost platform for the construction of microfluidic paper-based analytical devices ( $\mu$ PADs) in our work. The use of paper which is patterned with wax printing is a suitable method to manipulate 


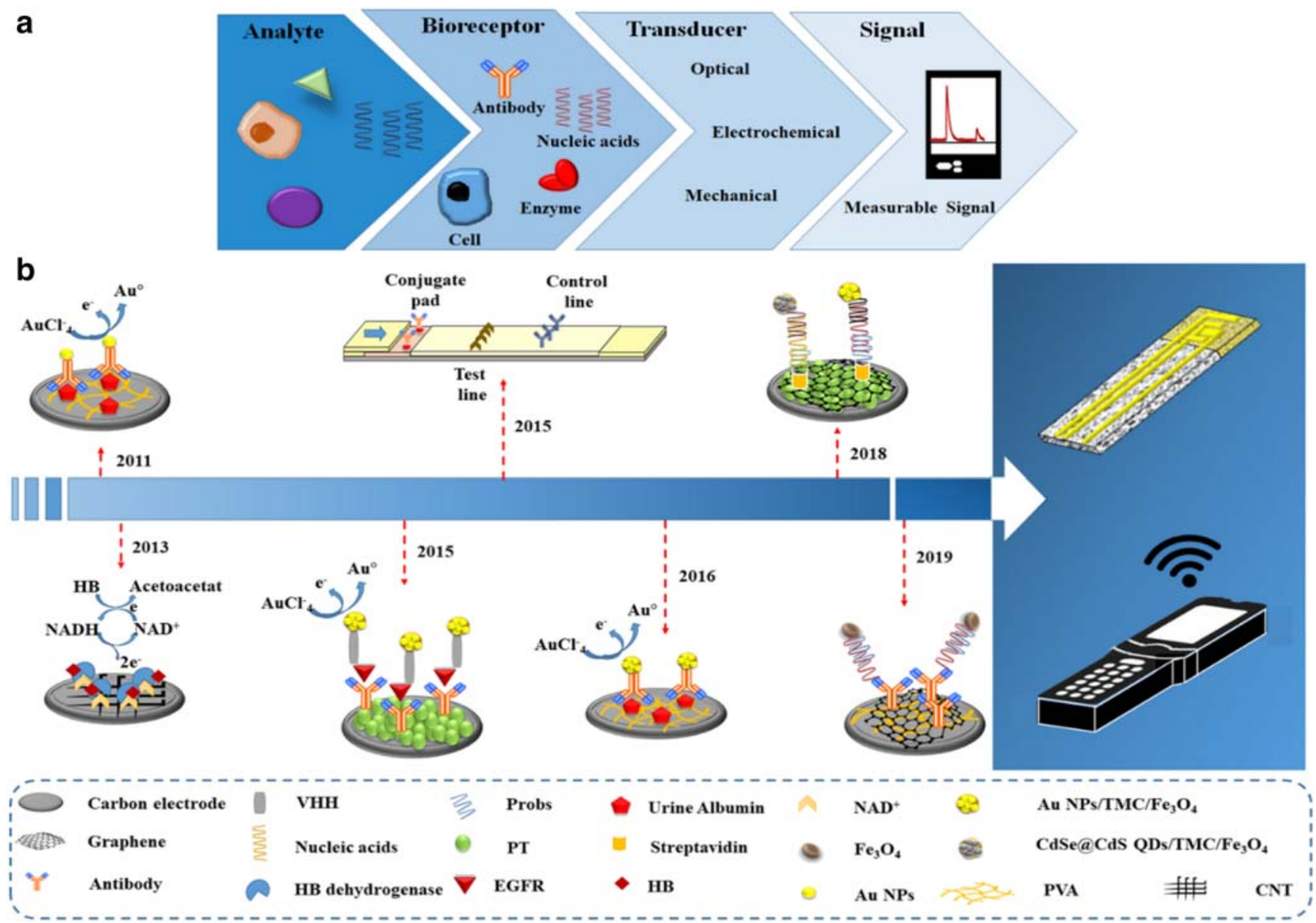

Fig. 1 Schematic representation of POC biosensor components (a), the path of POC biosensors development in our center based on paper and printed electrode technologies (b) and also our future outlook

liquid transport in the device. These devices have received considerable attention to become one of the important sensing methods for clinical diagnostics predominantly for POC testing applications [4]. A brief history of our developed biosensing devices is provided in Fig. 1 b.

\section{POC biosensors}

Two major types of POC biosensors are screen printing electrodes and paper-based microfluidic assay.

\section{Screen printing electrochemical POC biosensors}

Screen printing technology is one of the most extensively employed methods for large-scale production of cost effective and reliable electrochemical POC biosensors. This technology presents easy approaches to fabricate disposable POC instruments at large scales for real-time analysis or monitoring of a clinical biomarker [4].

\section{Lateral flow assay (LFA) based optical POC biosensors}

The capillary flow platform, also known as lateral flow assay (LFA), is a paper-based microfluidic platform for the determination of biomarkers and pathogens at the patient care and/or home use. This system presents a relatively fast and cost effective assay, which can be conducted by minimally trained personnel without extensive technical skills and sophisticated laboratory facility. These assays require small amounts of liquid sample and time of 5 to $20 \mathrm{~min}$ for producing a qualitative result in the presence or absence of the analyte [4].

In the following sections, examples of specific research subjects related to screen printing electrodes (electrochemical) and LFA (optical) POC biosensors, which were developed to detect clinical biomarkers, 
are discussed. Furthermore, some critical challenges that need to be surmounted in order to facilitate POCT translation into clinical practice, are presented.

\section{Screen printing (electrochemical) and LFA (optical) POC biosensors for protein biomarkers analysis}

Recognition of circulating protein biomarkers offers significant information for monitoring disease progression and treatment efficacy. The main analytical method for evaluating protein biomarkers is immunosensor, which is based on the protein/antigen-antibody reaction coupled to various label such as, nanoparticles, DNA barcodes, fluorescent and electrochemiluminescent labels to prepare the measurement of biomarker with high specificity and sensitivity. Several screen printing electrochemical and LFA POC biosensors have been developed in our center for high ultra-sensitive determination of protein biomarkers using different compounds at the nanoscale.

In 2011, Omidfar and coworkers reported a competitive electrochemical immunosensor for urine albumin detection using antibody conjugated gold nanoparticles (AuNPs) and polyvinyl alcohol modified screen-printed carbon electrode (SPCE). The proposed biosensor showed a linear response in the concentration range of 2.5 to $200 \mu \mathrm{g} / \mathrm{mL}$ and a limit of detection of $25 \mathrm{ng} / \mathrm{mL}$ [5]. Another urine albumin electrochemical biosensor was also developed in 2016 using Gold/Ntrimethyl chitosan/iron oxide $\left(\mathrm{Au} / \mathrm{TMC} / \mathrm{Fe}_{3} \mathrm{O}_{4}\right)$ nanocomposite labeled antibody. The tag was demonstrated to be capable of enhancing the detector signal by high-density assembly of gold particles on $\mathrm{TMC} / \mathrm{Fe}_{3} \mathrm{O}_{4}$ composite. This process was shown to be simple, reliable and capable of amplifying the recognition signal which potentially leads to decrease limit of detection $(0.2 \mathrm{pg} / \mathrm{mL})$ and dynamic range in comparison with previous work [6]. $\mathrm{Au} / \mathrm{TMC} / \mathrm{Fe}_{3} \mathrm{O}_{4}$ nanocomposite has also been employed as a tracing tag to label nanobody specific to epidermal growth factor receptor (EGFR). Due to the overexpression of EGFR in many aggressive cancer types, it is presented as a significant biomarker which provides this opportunity for early cancer diagnosis and thereby decreasing the morbidity and mortality associated with advanced disorder. The presented electrochemical biosensor showed a linear response in the range of concentration from 0 to $1000 \mathrm{pg} / \mathrm{mL}$ and a detection limit as low as $0.05 \mathrm{pg} / \mathrm{mL}$ [7].

We also designed three research subjects based on LFA, a well-established platform for optical POCT, using gold particles as a tracing tag and nitrocellulose membrane as a substrate platform for rapid measurement of protein biomarkers in real samples. In one operation for detecting human albumin in urine sample, nitrocellulose membrane was used without any treatment, and in another research subject, mesoporos silica was employed to modify the surface of the membrane in order to enhance the sensitivity of assay [8,9]. In 2015, Goudarzi et al., developed a new test strip assay for rapid detection serum specific immunoglobulin A (IgA) antibodies to Epstein-Barr virus viral capsid antigen. In this work, a conjugate of gold nanoparticle-anti humam IgA secondary antibody was employed as the detection probe [10].

\section{Screen printing electrochemical POC biosensors for nucleic acid biomarkers analysis}

Noninvasive measurement of circulating free nucleic acids (cfNAs) biomarkers including cell-free DNAs (cfDNA) and RNA in blood and other body fluids is of particularly importance for early detection and diagnosis of disease. We have developed several studies for ultra-sensitive detection of nucleic acid biomarkers using different materials at the nanoscale. Altered DNA methylation patterns have been recognized as one of the most common phenomenon in human cancers. In 2016, a chip based sandwich electrochemical genosensor has been established for the quantitative assessment of RASSF1A DNA promoter methylation using $\mathrm{Au} /$ $\mathrm{TMC} / \mathrm{Fe}_{3} \mathrm{O}_{4}$ nanocomposite as tracing tag to label DNA probe and polythiophene (PT) as immobilization support of electrode surface. This system can detect DNA methylation in the nano-molar to the pico-molar range with a limit of detection down to $2 \times 10^{-15} \mathrm{M}$ [11]. Recently, another electrochemical genosensor was described for early detection of circulating methylated DNA (E-cadherin) using ssDNA probe conjugated to $\mathrm{Fe}_{3} \mathrm{O}_{4}$-citric acid nanocomposites and antibody against 5-methylcytosine which was physically immobilized onto the reduced graphene oxide and polyvinylalcohol modified electrode. The developed biosensor can detect circulating methylated DNA with a wide dynamic range from $1 \times 10^{-4}$ to $20 \mathrm{ng} / \mathrm{mL}$ and sensitivity down to $9 \times 10^{-5} \mathrm{ng} / \mathrm{mL}$ [12] .

miRNAs are one of the novel biomarkers that could be applied to detect cancer in early stage. In a study by Daneshpour et al., a sandwich based electrochemical genosensor was established for the ultrasensitive detection of microRNA (miR-106a) using a double-specific probe procedure and $\mathrm{Au} / \mathrm{TMC} / \mathrm{Fe}_{3} \mathrm{O}_{4}$ nanocomposites as tracing tag. This system had a linear relationship ranging from $1 \times 10^{-3} \mathrm{pM}$ to $1 \times 10^{3} \mathrm{pM}$ and a detection limit around $3 \times 10^{-4} \mathrm{pM}$ [13]. In 2018, this group can develop an electrochemical genosensor to recognize two cancer related miRNAs simultaneously by using Au nanoparticles and CdSe@CdS quantum dotscontained magnetic nanocomposite as tracing tags along with the polythiophene/reduced graphene oxide-modified electrodes. The presented POC system showed the detection limit around $0.02 \mathrm{fM}$ and $0.06 \mathrm{fM}$ for let-7a and miR-106a, respectively [14]. 


\section{Screen printing electrochemical POC biosensors for metabolite biomarkers analysis}

Low molecular weight metabolites, often called small molecules, are intermediate or end products of metabolic reactions that catalyzed by an enzyme and occur naturally within cells. Quantitative determination of metabolites as biomarkers are valuable in: i) making a diagnosis, assessing its severity and treatment effectiveness, ii) identifying risk biomarkers for forecasting future improvement of diseases. $\beta$ Hydroxybutyric acid, also known as 3-hydroxybutyric acid, is one of the main blood ketone and considered as a key biomarker for management of diabetic ketoacidosis. In 2013, one study for detecting 3-hydroxybutyrate (HB) was developed in our group based on single-walled carbon nanotubes (SWCNTs) modified SPCE. In this study, HB dehydrogenase as a recognition element was physically immobilized on the SWCNTs surface, followed by the addition of $\mathrm{NAD}^{+}$and target, the current of the system changes through the oxidation reaction of $\mathrm{NAD}^{+}$to $N A D H$. This sensitive biosensor exhibited a linear range from 0.1 to $2 \mathrm{mM} \mathrm{pg} / \mathrm{mL}$ with a limit of detection as low as $0.009 \mathrm{mM}$ [15]. This group has also developed another HB dehydrogenase POC system, that SWCNT was employed to immobilize the cofactor $\mathrm{NAD}^{+}$ on the surface of SPCE. This system was capable of detecting HB with a linear range of $0.01 \mathrm{mM}$ to $0.1 \mathrm{mM}$ and a low detection limit of $0.009 \mathrm{mM}$ [16].

Recent studies have demonstrated that $\mu \mathrm{PA}$ devices has attracted increasing attention for various biomarker detection $[1,2]$. So, in two ongoing research subjects, we used cellulose nanofiber membranes as a supporting matrix and also as an immobilizing platform for fabricating $\mu \mathrm{PA}$ devices in order to detect metabolite and protein biomarkers.

\section{Conclusion and future outlook}

This report summarizes our studies in recent years, referring to electrochemical and optical biosensors based POCT for detection of disease biomarkers ranging from proteins to nucleic acids and metabolite. In the fabrication of electrochemical genosensor and immunosensor, disposable SPCEs composed of three electrode system (working, counter and reference electrodes) were extensively applied as electrode substrate. Various nanomaterials including AuNPs, $\mathrm{Fe}_{3} \mathrm{O}_{4} / \mathrm{Au}$ coreshell nanoparticle, graphene oxide, and quantum dots were successfully incorporated into electrode matrixes to enhance the assay sensitivity. In addition to SPEs, paper based electrodes was also employed to detect targets of interest. Paperbased electrochemical systems provide ideal alternative approach for affordable diagnostic applications due to their small sizes, easy fabrication, and cost effectiveness.
Although several biosensors based POCT have been developed for the detection of various analysts, the certain crucial issues and challenges need to be addressed before the practical use in the clinic and wide-scale production. These limitations include the following: (i) inadequate detection sensitivity to distinguish biomarkers at the different stages of the diseases in various samples with a cost-effective manner to take adequate clinical management and improve patient treatment, (ii) high selectivity and multiplexed capacity (detection of biomarkers at different clinical ranges and molecular levels) are required to develop diagnostic strategies within a single test. Considering the above-mentioned challenges, our future research will be extended to overcome such limitations which would greatly enhance the development of biomarkerdetecting POC biosensors. In the upcoming investigates, more and deeper studies will be dedicated to reducing batch-tobatch variations, fabricating multiplexed sensing platform and finally utilizing novel nanomaterials and substrates to develop high sensitive and biocompatible devices for monitoring patient health at anywhere and anytime.

Acknowledgments If the achievements of this center are successful, I owe gratitude to my students, researchers and colleagues for their wonderful collaboration in the past and present. Many of them appear in this paper; but of those who don't, let me mention them here: Hanieh shirazi, Maryam Daneshpour, Shima Kabiri, Fahimeh Khorsand, Maedeh Darzianiazizi, Nahid Shoaee, Ahmad Dehdast, and Zahra Mirzaiezadeh.

\section{Compliance with ethical standards}

Conflict of interest The authors declared that they have no conflict of interest.

\section{References}

1. Syedmoradi L, Ahmadi A, Norton ML, Omidfar K. A review on nanomaterial-based field effect transistor technology for biomarker detection. Microchim Acta. 2019;186(11):739.

2. Ahmadi A, Kabiri S, Omidfar K. Advances in HbA1c biosensor development based on field effect transistors: a review. IEEE Sensors J 2020

3. Carter LJ, Garner LV, Smoot JW, Li Y, Zhou Q, Saveson CJ et al. Assay techniques and test development for COVID-19 diagnosis. ACS Publications; 2020.

4. Syedmoradi L, Daneshpour M, Alvandipour M, Gomez FA, Hajghassem H, Omidfar K. Point of care testing: the impact of nanotechnology. Biosens Bioelectron. 2017;87:373-87.

5. Omidfar K, Dehdast A, Zarei H, Sourkohi BK, Larijani B. Development of urinary albumin immunosensor based on colloidal AuNP and PVA. Biosens Bioelectron. 2011;26(10):4177-83.

6. Shirazi H, Ahmadi A, Darzianiazizi M, Kashanian S, Kashanian S, Omidfar K. Signal amplification strategy using gold/N-trimethyl chitosan/iron oxide magnetic composite nanoparticles as a tracer tag for high-sensitive electrochemical detection. IET nanobiotechnology. 2016;10(1):20-7.

7. Omidfar K, Darzianiazizi M, Ahmadi A, Daneshpour M, Shirazi H. A high sensitive electrochemical nanoimmunosensor based on $\mathrm{Fe} 3 \mathrm{O} 4 / \mathrm{TMC} / \mathrm{au}$ nanocomposite and PT-modified electrode for the 
detection of cancer biomarker epidermal growth factor receptor. Sensors Actuators B Chem. 2015;220:1311-9.

8. Omidfar K, Kia S, Larijani B. Development of a colloidal goldbased immunochromatographic test strip for screening of microalbuminuria. Hybridoma. 2011;30(2):117-24.

9. Omidfar K, Khorsand B, Larijani B. Development of a new sensitive immunostrip assay based on mesoporous silica and colloidal au nanoparticles. Mol Biol Rep. 2012;39(2):1253-9.

10. Goudarzi S, Ahmadi A, Farhadi M, Kamrava SK, Mobarrez F, Omidfar K. A new gold nanoparticle based rapid immunochromatographic assay for screening EBV-VCA specific IgA in nasopharyngeal carcinomas. J Appl Biomed. 2015;13(2): 123-9.

11. Daneshpour M, Izadi P, Omidfar K. Femtomolar level detection of RASSF1A tumor suppressor gene methylation by electrochemical nano-genosensor based on $\mathrm{Fe} 3 \mathrm{O} 4 / \mathrm{TMC} / \mathrm{au}$ nanocomposite and PTmodified electrode. Biosens Bioelectron. 2016;77:1095-103.

12. Khodaei R, Ahmady A, Khoshfetrat SM, Kashanian S, Tavangar SM, Omidfar K. Voltammetric immunosensor for E-cadherin promoter DNA methylation using a Fe $3 \mathrm{O}$ 4-citric acid nanocomposite and a screen-printed carbon electrode modified with poly (vinyl alcohol) and reduced graphene oxide. Microchim Acta. 2019;186(3):170.

13. Daneshpour M, Omidfar K, Ghanbarian H. A novel electrochemical nanobiosensor for the ultrasensitive and specific detection of femtomolar-level gastric cancer biomarker miRNA-106a. Beilstein journal of nanotechnology. 2016;7(1):2023-36.

14. Daneshpour M, Karimi B, Omidfar K. Simultaneous detection of gastric cancer-involved miR-106a and let-7a through a dual-signalmarked electrochemical nanobiosensor. Biosens Bioelectron. 2018;109:197-205.

15. Khorsand F, Riahi S, Fard SE, Kashanian S, Naeemy A, Larijani B, et al. Development of 3-hydroxybutyrate dehydrogenase enzyme biosensor based on carbon nanotube-modified screen-printed electrode. IET nanobiotechnology. 2013;7(1):1-6.

16. Khorsand F, Azizi MD, Naeemy A, Larijani B, Omidfar K. An electrochemical biosensor for 3-hydroxybutyrate detection based on screen-printed electrode modified by coenzyme functionalized carbon nanotubes. Mol Biol Rep. 2013;40(3):2327-34.

Publisher's note Springer Nature remains neutral with regard to jurisdictional claims in published maps and institutional affiliations. 